\title{
Using Evolutionary Algorithms and Dynamic Programming to solve Uncertain Multi-Criteria Optimization Problems with application to Lifetime Management for Military Platforms
}

\author{
Claire J. Thie \\ CSIP, QinetiQ Ltd \\ Malvern Technology Centre \\ Malvern, Worcestershire, \\ WR14 3PS, UK \\ cthie@signal.qinetiq.com
}

\author{
Darren M. Chitty \\ CSIP, QinetiQ Ltd, \\ Malvern Technology Centre, \\ Malvern, Worcestershire, \\ WR14 3PS, UK \\ Colin M. Reed \\ CSIP, QinetiQ Ltd, \\ Malvern Technology Centre, \\ Malvern, Worcestershire, \\ WR14 3PS, UK \\ dchitty@signal.qinetiq.com creed@signal.qinetiq.com
}

\begin{abstract}
Microelectronics are typically critical components in a military platform, some of which may become obsolete, before the equipment life cycle end. Obsolete components may be required for a number of reasons. Components can become obsolete even before production of a platform commences. The selection of solutions for resolving obsolete components throughout a platform can be considered as a multi-criteria optimization problem where the aim is to select the most cost effective solutions for resolving a portfolio of obsolescence arisings. In this paper we consider the case where the criteria with which the options are evaluated are not point values, but probability distributions generated by a Bayesian Belief Network. We propose the use of an evaluation technique called measures of effectiveness (MOE), that can capture and use the probabilistic information associated with potential solutions. This is used with two candidate optimization techniques, Dynamic Programming (DP) and Evolutionary Algorithms (EAs), to identify cost effective solutions for resolving obsolescent components throughout a platform. Both optimization techniques were able to identify a number of solutions at different cost and MOE levels; the solutions that form the DP Pareto front dominate very slightly in places those that form the EA Pareto front.
\end{abstract}

\section{Categories and Subject Descriptors}

C.4 [Performance of Systems]: Reliability, Availability, Serviceability; G.1.6 [Optimization]: Constrained Optimization; G.7 [Computers in Other Systems]: Military

\section{General Terms}

Algorithms, reliability

Permission to make digital or hard copies of all or part of this work for personal or classroom use is granted without fee provided that copies are not made or distributed for profit or commercial advantage and that copies bear this notice and the full citation on the first page. To copy otherwise, to republish, to post on servers or to redistribute to lists, requires prior specific permission and/or a fee.

GECCO'05, June 25-29, 2005, Washington, DC, USA.

Copyright 2005 ACM 1-59593-097-3/05/0006 ...\$5.00.

\section{Keywords}

multi-criteria, measures of effectiveness, uncertainty

\section{INTRODUCTION}

Microelectronics are typically critical components in a military platform, some of which may become obsolete, before the equipment life cycle end. Obsolete components may be required for a number of reasons. Components can become obsolete even before production of a platform commences. This problem has been recognized by the defence industry for some time.

The production of a set of solutions to a portfolio of obsolete microelectronics components across the whole of a complex system or platform can be considered as a multi-criteria optimization problem. Each obsolete component will have a number of options that may be used to resolve the $O b$ solescence Arising (OA), such as a Last Time Buy of components, or a redesign of the circuit boards containing the obsolete component. Each option for resolving an obsolete component will have a number of criteria (such as acquisition costs, reliability, system availability, time to implement) associated with it that may be used to evaluate the costeffectiveness of that option. The problem is a complex one and requires sophisticated optimization techniques that can be used to maximize the beneficial criteria and minimize the costs across all of the options selected. These are not likely to be wholly complimentary and so a trade-off of the costs against the benefits may be required. Techniques for evaluating a particular option, in the multi-criteria domain, will typically split the criteria into 'costs' and 'benefits', and then aggregate the values for these criteria into a single cost and a single benefit value. Typically the decision process needs to weight the criteria according to their relative importance, and the aggregation consists of a weighted sum of the criteria values. Such problems are referred to as multi-objective as there is more than one objective function to optimize against. The Pareto optimal set is a set of solutions each of which are not dominated by the others in terms of all criteria. Thus the optimal solution in multi-objective problems is to produce the Pareto optimal set. These are presented to a decision maker to make the final choice that provides a desirable trade-off. The traditional optimization technique 


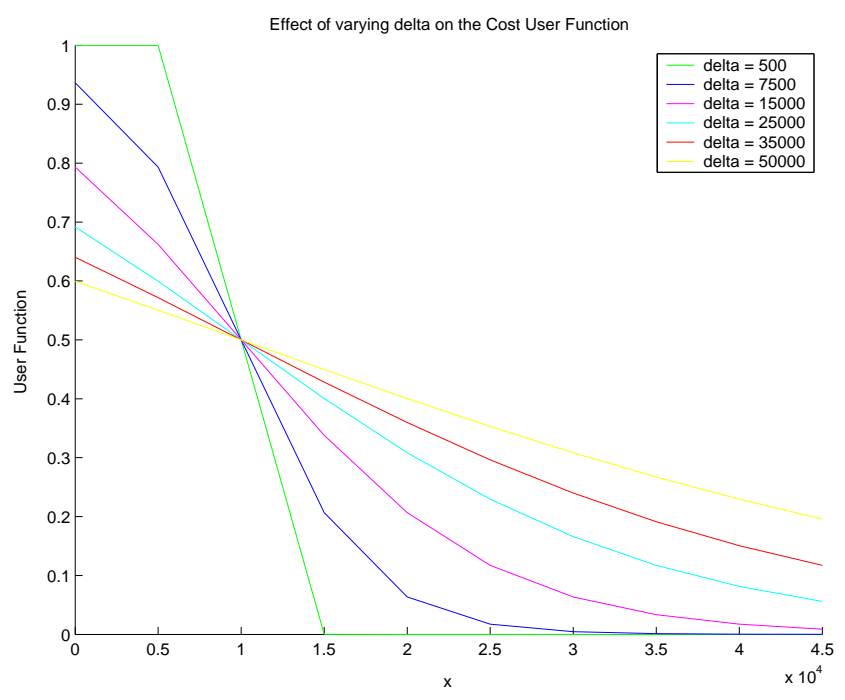

Figure 1: An example of a user function for a cost criterion with changing slope angle.

Dynamic Programming (DP) [1] can be used to obtain a Pareto optimal set of solutions for a two (conflicting) criteria problem by choosing options that maximize benefits for a number of increasing cost bands. Evolutionary Algorithms (EAs) are particularly suitable in the solution of multi-objective optimization problems, as at each iteration they maintain a population of potential solutions and hence can be used to find multiple trade-off solutions [2]. EAs can be used to obtain solutions to true multi-objective optimization problems where each of the individual cost and benefit criteria are kept separate. The problem considered here, however, is further complicated through the presence of uncertain criteria values.

\section{UNCERTAIN CRITERIA VALUES}

A Bayesian Belief Network (BBN) is a technique that allows reasoning to be carried out in uncertain circumstances [5]. For this work, the uncertainty and dependencies associated with the criteria that are used to assess the various options for dealing with an obsolete component have been modelled using a BBN. In order to assess the 'value' of a particular option, the available evidence is propagated through the network to produce a probability distribution embodying the likelihood of particular values for each of the criteria. One method to deal with this issue could be to take the average value of the probability distribution for each of the criteria, and proceed with the evaluation methodology described above. However, this has the disadvantage that we will essentially be throwing away useful information that has been assimilated by the BBN. An alternative method that can capture and use all the information available and be used as an evaluation technique is more desirable.

Measures of Effectiveness (MOE) measure the extent to which system outputs satisfy related user requirements (see [6] for more details); in this case suitable outputs would be actual cost compared to budget, system availability, and implementation time scales. Their formulation is applicable to any data type, and so can deal with system characteristics expressed as probability density functions. MOEs can also take account of dependencies between criteria. In addition, multiple, even conflicting user requirements can be accounted for. MOEs are a measure of how the observed values for criteria match with the users' requirements. This is achieved through the use of a user function which returns values between zero and one quantifying acceptability, with zero being least acceptable and one being most acceptable, the range of possible values that a criteria may take. For example a stakeholder may consider a cost of zero dollars to be the most acceptable and a cost of one million dollars and above to be unacceptable to pay for the design of a new component. These two design costs would be allocated the maximum and minimum values respectively by the user function, and the user function values assigned to the design costs in between would decrease monotonically at a rate specified by the stakeholder. The user function for the acceptability of cost used in this work is specified by:

$$
f(x)=1-0.5(1+\tanh (a(x-b)))
$$

where parameter $b$ is the value of $x$ for $f(x)=0.5$, and the parameter $a$ sets the curve shape through the following equation:

$$
\Delta=\frac{1.099}{a}
$$

where $\Delta$ is the separation in $x$ between $f(x)=0.5$ and $f(x)=0.1$ points. By changing the value of $\Delta$ we alter the mid-slope of the curve that determines the rate at which the users' acceptability of the criteria under consideration changes. As can be seen in Figure 1, smaller values of $\Delta$, produce steeper slopes, whilst larger values produce shallower slopes. Similarly a benefit user function is the compliment of the cost function. An obsolescence arising may have more than one location. This may result in locations having different user functions for each of the criteria to reflect differing priorities. The MOE framework employs the user function information and the BBN outputs for each criteria to produce an MOE value for each criterion that reflects the degree to which the stakeholders' requirements have been met. The MOE values for each of the criterion for each option can be combined to produce a single MOE for a set of solutions, as is required here. The MOE values are combined in this way to facilitate their display. The potential sets of solutions produced by each of the optimization techniques can then be plotted against their acquisition costs in order that an appropriate set of solutions be selected.

\section{INITIAL EXPERIMENTS}

In order to evaluate the use of both DP and EA with MOE some initial experiments were carried out using a hypothetical scenario. The example problem to be considered is a platform that consists of three systems: radar; flight computer; digital engine control unit. Each system has two circuit boards. The problem scenario consists of a Last Time Buy (LTB) notice for obsolete component IC5 (part type E) on one of the boards in the radar. A LTB notice is a typical OA trigger although it is only one of a number of resolutions. For each component in the platform, information on the number of components (and their types) used on each board, and the number of years until obsolescence is available. Another factor when determining a resolution is to take account of other current or predicted OAs; analysis of all three systems in the platform reveals further obsolete components that affect five out of the six boards. When considering a resolution account also needs to be taken of planned activities such as technology refresh and planned re- 


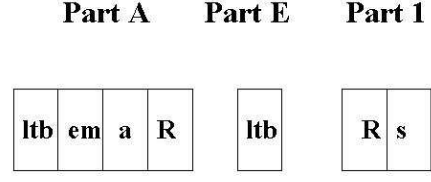
Board
$\begin{array}{llll}1 & 2 & 3 & 5\end{array}$
1
56

Figure 2: The representation of a potential solution, where 'ltb' indicates a last time buy option has been chosen, 'em' indicates that the emulation option has been chosen, ' $a$ ' indicates that the alternate option has been chosen, ' $s$ ' indicates that the substitute option has been chosen, and ' $R$ ' indicates that a board redesign option has been chosen.

designs. Further analysis of the platform reveals that a further four part types will become obsolete before the boards on which they are placed are due to be upgraded. This provides an example portfolio of seven OAs and potential OAs to be resolved. The format of a potential solution is given in figure 2. It illustrates a suite of potential solutions for a three component, six board obsolescence problem. Part type A can be found on boards 1,2,3, and 5, part type E can be found on board 1 and part type 1 can be found on boards five and six. The options are available at both the component and board level. If a board level option is chosen for a part type, then that option must be specified for every other component that is placed on that board. For example, in the above solution representation, a board redesign has been specified for part type A and part type 1 for board 5. This solution representation was used by both the DP and EA optimization techniques in a prototype optimization tool.

The prototype optimization tool was evaluated on two scenarios. The first scenario consisted of a two-criteria problem with each option for resolving an OA having a single cost and a single benefit associated with it. The second scenario had multiple costs and multiple benefits associated with each option. Both scenarios make use of user functions with a medium slope and each cost and benefit is described by a probability distribution. Each of these values are assumed to be normally distributed and are dummy values that have been assigned by non-experts. In an operational tool, these values would be obtained from the BBN. The EA used two populations containing 20 individuals each (one to maximise beneficial criteria and one to minimise cost criteria) and was run for 100,000 generations for both scenarios. The separate populations exchanged randomly selected individuals every ten generations. Both optimization techniques were able to identify a number of solutions at different cost and MOE levels for both scenarios, but the solutions that form the DP Pareto front dominate (outperform in terms of cost and benefit) very slightly in places those that form the EA Pareto front. This may be because the EA has not been allowed to carry out sufficient generations of its search. It should be noted that the example problems tackled during the project described here are relatively small and hence the optimization algorithms take only seconds to run. However, dynamic programming is a computationally intensive technique that may not scale well as the problem sizes increase. Whilst steps can be taken to constrain the size of the solution space that is to be explored, it may be impractical or impossible to use for many real world problems where solu- tions are required in a short amount of time. Therefore, the next-best-thing is to obtain a reasonable approximation to the solution. This can be achieved by enhancing the search strategy by using a heuristic technique such as an EA to make it more informed and search only the promising areas of the search space. Both of the proposed techniques now need to be tested on real scenarios.

This work forms part of an ongoing MOD-funded research project which aims to develop a decision-aide tool to enable users to identify optimum system-wide obsolescence solutions in complex systems, to be published separately [4]. QinetiQ has also performed work to address alternative interpretations of the term obsolescence [3]. In particular, it has developed underlying concepts related to the obsolescence of systems such as trains or aircraft, recognizing that this can be affected by the full range of PESTLE (political, economic, social, technical, legislative, environmental) factors. A method for system lifetime management has been derived and validated by application to a number of large systems. It is planned that the method will be refined by exploiting a variety of optimization techniques.

\section{CONCLUSIONS}

This paper describes the use of MOE in combination with DP and EA in order to identify potential solutions for resolving a portfolio of obsolescence arisings for military platforms. MOEs offer a practical method for the evaluation of options using probabilistic criteria values. In initial experiments using a hypothetical scenario, DP produced solutions that dominated those produced by the EA in terms of cost and MOE value. However, DP may not scale well as the problem size increases, as it is a computationally intensive technique. In this case, EAs could provide good, but not necessarily optimal, solutions in a reasonable time. This will require further investigation. The optimization tool is currently being incorporated into a tool for managing uncertainty in microelectronics obsolescence to be tested by potential users in order to ensure the desired functionality is achieved and that the proposed techniques scale well. This will involve testing the tool with real scenarios.

\section{ACKNOWLEDGMENTS}

This work has been funded by the UK Ministry of Defence's Corporate Research Programme. The authors would like to thank Mike Housley, Ted Smith and Steve Grist for their support and advice during this programme of work.

\section{REFERENCES}

[1] D. P. Bertsekas. Dynamic Programming and Optimal Control. Athena Scientific, 1995.

[2] K. Deb. Multi-Objective Optimization using Evolutionary Algorithms. John Wiley, 2001.

[3] T. Dowling. Technology insertion and obsolescence. Journal of Defence Science, 9(3), 2004.

[4] M. Housley. To be presented at the COG International Conference, June 2005.

[5] J. Pearl. Probabilistic Reasoning in Intelligent Systems: Networks of Plausible Inference. Morgan Kauffman, 1988.

[6] C. Reed and A. Fenwick. A consistent multi-user, multi-goal framework for assessing system performance with application to a sonar system. Submitted to USN Journal of underwater Acoustics, 2005. 